\title{
METODOLOGÍAS ACTIVAS PARA LA ENSEÑANZA Y EL APRENDIZAJE
}

\author{
Ma. del Carmen Bernal González
} Mariel Sarai Martínez Dueñas

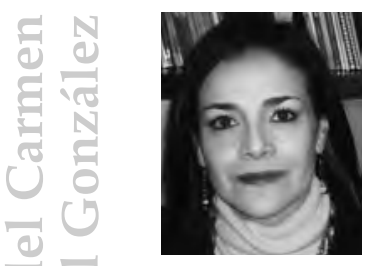

Licenciada en Pedagogía, Universidad Panamericana, México. Doctora en Ciencias de la Educación, Universidad de Navarra, España. Fue directora de la Facultad de Pedagogía de la Universidad Panamericana. Entre sus líneas de investigación se encuentran: educación estética; teoría pedagógica; proyecto educativo vasconcelista. Entre sus publicaciones y trabajos de investigación: La teoría pedagógica de José Vasconcelos, Trillas, México; coautora de Educatividad y educabilidad, Minos Tercer Milenio/UP, México. Miembro del Consejo Técnico del Examen General de Egreso de las Licenciaturas en Pedagogía y Ciencias de la Educación, CENEVAL. Miembro del Consejo de Rectoría de la Universidad Panamericana. Miembro del Consejo Editorial de la revista Saberes y Quehaceres del Pedagogo. Miembro del Consejo Científico de la Revista ESE, estudios sobre educación, Universidad de Navarra, España. Miembro del Colegio de Pedagogos de México.

Correo electrónico: [mbernal@up.edu.mx].

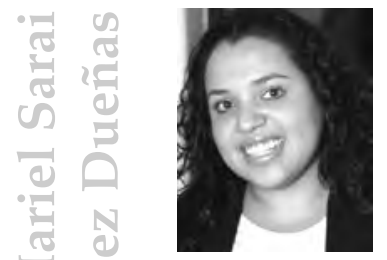

Licenciatura en Pedagogía, Universidad Panamericana. Maestría en Matrimonio y Familia, Universidad de Navarra, España. Coordinadora de la Licenciatura en Pedagogía, Facultad de Pedagogía de la Universidad Panamericana. Colabora con el departamento de innovación educativa y admisiones de la Facultad de Pedagogía de la Universidad Panamericana. Temas de interés: tecnología educativa; desarrollo de recursos pedagógicos; orientación educativa, vocacional y profesional; diagnóstico psicométrico; adolescencia; estudio interdisciplinar de la familia. Participante en conferencias y medios de comunicación con temas de familia, juventud, adolescencia, violencia intrafamiliar, orientación vocacional, posmodernidad y bullying.

Correo electrónico: [msmartin@up.edu.mx]. 
Lo que hace el estudiante es, en realidad, más importante para determinar lo que aprende, que lo que hace el profesor. Thomas J. Shuell

Las personas aprendemos de manera natural. Sin embargo, enseñar se ha vuelto una tarea cada vez más exigente para quienes ejercen la docencia en los diferentes niveles. La experiencia dicta, en palabras de Mark Prensky, que «hoy tenemos alumnos para los que nuestro sistema educativo no fue creado» (Prensky, 2001, 1). Lo anterior manifiesta la urgencia de innovar la labor docente y encontrar mejores metodologías para hacer asequible el aprendizaje a los estudiantes.

Vivimos en la era de la diversificación. Desde la década de 1990, las aulas no se volvieron más grandes sino más diversificadas en cuanto a las capacidades, motivación y base cultural de los estudiantes. Los modelos de clase magistral y tutoría tradicional perdieron vigencia en clases en donde era cada vez más complejo mantener un alto nivel académico. Paulatinamente se fue asimilando que la enseñanza de calidad consistía en estimular a los estudiantes para utilizar los procesos de aprendizaje de forma espontánea, cobrando así relevancia el papel del estudiante por encima del papel del profesor (López, 2002, 9).

Lo anterior nos remite a lo que hoy se conoce como «metodologías activas» que, en esencia, retoman tres ideas principales:

1. El estudiante es un protagonista activo de su aprendizaje.

2. El aprendizaje es social. Los estudiantes aprenden mucho más de la interacción que surge entre ellos que solamente de la exposición.

3. Los aprendizajes deben ser significativos. El aprendizaje requiere ser realista, viable y complejo de forma que el estudiante halle relevancia en la transferencia de dicho contenido.

Para que se produzca un aprendizaje significativo, deben producirse dos condiciones: 
a) Presentación de un material potencialmente significativo. Lo anterior implica que posee un significado lógico, es decir, relacionado de forma sustantiva con la estructura cognitiva de quien aprende. Además, un material potencialmente significativo cuenta con ideas anclaje, que facilitan la interacción con el sujeto que aprende.

b) Actitud potencialmente significativa de aprendizaje por parte del educando. Implica que se tiene la voluntad del educando para aprender.

Los aprendizajes significativos guardan suma relevancia en las metodologías activas de aprendizaje, pues los estudiantes -hoy más que nunca- buscan que lo aprendido guarde relación con lo previamente aprendido, pero sobre todo que sea un contenido relevante, comprobable y con aplicación en la vida diaria.

Aun cuando el estudiante es el protagonista de su aprendizaje, requiere de la función directiva del profesor, quien no sólo presentará la cultura y el aprendizaje en general de forma atractiva, sino que además deberá conseguir que, quien aprenda, «construya» sus contenidos y los transforme en aprendizajes. Cabe destacar que las metodologías activas juegan un papel relevante para lograr dicho cometido.

Las metodologías activas promueven, principalmente, dos características del aprendizaje:

1. Sociabilidad del aprendizaje. Para que una persona aprenda, debe lograr la interacción con otros; fomentar y favorecer el diálogo e intercambio de ideas.

2. Interactividad del aprendizaje. El uso de las nuevas tecnologías ayuda a la generación de comunidades de aprendizaje, facilitando la interacción y trascendiendo las barreras del tiempo y la distancia.

Hoy existe gran diversidad de metodologías activas. Conocer su fundamento permite a los profesores identificarlas y seleccionar aquéllas más a fines a los estudiantes y a los contenidos. 
A continuación se describen algunas metodologías activas ${ }^{1}$ :

- Aprendizaje cooperativo. Es el proceso de aprender en grupo y en comunidad. Pretende la formación de comunidades de aprendizaje, entendidas como un grupo de personas que, reunidas en un lugar y tiempo determinados, se ocupan de una tarea que les exige asumir funciones específicas e interactuar para el logro de una meta en común.

- Enfoque por competencias. Dentro del ámbito académico, «las competencias se definen como las acciones que el alumno deberá ser capaz de efectuar después del aprendizaje» (Corominas, Tesouro, Capell, Teixidó, Pélach y Cortada, 2006: 303). Lo anterior implica que primero se lleva cabo un proceso de aprendizaje y posteriormente se aplica; es en dicha acción en la que se verifica el nivel de desarrollo de la competencia.

- Aprendizaje basado en problemas. Consiste en un enfoque inductivo en el que los estudiantes aprenden el contenido de la sesión a través de la resolución de problemas reales.

- Método del caso. Se fundamenta en el análisis de una situación real o hipotética a través de una discusión dirigida, en la que se pretende conocer la diversidad de opiniones y favorecer el intercambio de experiencias.

- Enfoque cerebral del aprendizaje o "cerebro que aprende». Hace referencia a las bases neurofisiológicas del aprendizaje. Implica ponderar la estructura cerebral así como su influencia en el aprendizaje. Concentra todas aquellas teorías sobre la estimulación de los procesos mentales.

- Interactividad del aprendizaje. El concepto de interactividad es inherente a los procesos de comunicación, pues para que exista

1 Al final del documento se sugieren algunas fuentes de consulta para profundizar aún más. 
comunicación es imprescindible la interacción entre los participantes (ya sean personas, o bien surgir entre personas y cualquier dispositivo de las tecnologías de la información y la comunicación). Esta metodología, favorece la relación en comunidades de aprendizaje de forma asíncrona.

- Aprendizaje basado en la experiencia. Consiste en aprender mediante experiencias pasadas; también se le conoce como «aprendizaje basado en el error»: el protagonista del aprendizaje se encarga de obtener «experiencia» de sus errores.

- Práctica reflexiva. Se basa en el trabajo en grupos dirigidos por un experto que promueve la reflexión a partir de alguna experiencia. A menudo la reflexión se basa en ciertas estrategias como, el diario reflexivo (descripción/ reflexión/transferencia), y suele incluir la elaboración de un portafolios de evidencias.

- Simulación. El aprendizaje se muestra a través de la dramatización de situaciones hipotéticas. La riqueza de esta metodología radica en la reflexión póstuma y en la mediación del moderador.

Para concluir, puede afirmarse que la clave del éxito en el desarrollo de las metodologías activas consiste en tener presente que lo más importante es el alumno, cada alumno. La idea de que los estudiantes sean estudiantes felices, bien desarrollados, libres de progresar a su ritmo y que adquieran más fácilmente los conocimientos fundamentales, no es una utopía, debe ser una realidad. El papel de los profesores en el éxito del aprendizaje radica en el compromiso que tienen al desarrollar una profesión que aman y que los motiva, pues se sienten libres para desarrollar su propia manera de enseñar.

Afirma Thomas J. Shuell:

Para que los estudiantes consigan los resultados deseados de una manera razonablemente eficaz, la tarea fundamental del profesor consiste en 
lograr que sus alumnos realicen las actividades de aprendizaje que, con mayor probabilidad, les lleven a alcanzar los resultados pretendidos.

\section{BIBLIOGRAFÍA}

BIGGS, J., Calidad del aprendizaje universitario, Narcea, Madrid, 2005.

COLL; MARTIN; MAURI; MIRAS; ONRUBIA; SOLÉ y ZABALA, El constructivismo en el aula, Grao, España, 2005.

DANIELS, H., Vygotsky y la pedagogía, Paidós, España, 2003.

FERREIRO, G. y CALDERÓN, E., El ABC del aprendizaje cooperativo, Trillas, México, 2002.

JENSEN, E., Cerebro y aprendizaje, Narcea, Madrid, 2004.

LÓPEZ y LEAL, Cómo aprender en la sociedad del conocimiento, Gestión, España, 2000.

PRESNSKY, M., «Digital natives, digital inmigrants», On th Horizon, MCB University Press, USA, 2001.

SAINT, M., Yo explico pero ellos... ¿aprenden?, Mensajero, España, 2000.

VILLALOBOS, M., Evaluación del aprendizaje basado en competencias, Minos, México, 2009. 